\title{
Extremal solutions for $p$-Laplacian fractional integro-differential equation with integral conditions on infinite intervals via iterative computation
}

Ying Wang ${ }^{1,2}$, Lishan $\mathrm{Liu}^{1,3^{*}}$ and Yonghong $\mathrm{Wu}^{3}$

"Correspondence:

mathlls@163.com

'School of Mathematical Sciences,

Qufu Normal University, Qufu,

Shandong 273165, People's

Republic of China

${ }^{3}$ Department of Mathematics and

Statistics, Curtin University of

Technology, Perth, WA 6845,

Australia

Full list of author information is available at the end of the article

\begin{abstract}
We study the extremal solutions of a class of fractional integro-differential equation with integral conditions on infinite intervals involving the $p$-Laplacian operator. By means of the monotone iterative technique and combining with suitable conditions, the existence of the maximal and minimal solutions to the fractional differential equation is obtained. In addition, we establish iterative schemes for approximating the solutions, which start from the known simple linear functions. Finally, an example is given to confirm our main results.
\end{abstract}

MSC: $34 \mathrm{~B} 18 ; 34 \mathrm{~B} 40$

Keywords: extremal solutions; monotone iterative method; fractional differential equation; $p$-Laplacian operator; infinite intervals

\section{Introduction}

In this paper, we study the existence of extremal solutions to the following fractional integro-differential equation with $p$-Laplacian operator on infinite intervals:

$$
\left\{\begin{array}{l}
D_{0^{+}}^{\beta}\left(\varphi_{p}\left(D_{0^{+}}^{\alpha} x(t)\right)\right)+a(t) f(t, x(t),(T x)(t),(S x)(t))=0, \quad t \in J^{\prime}, \\
x(0)=x^{\prime}(0)=\cdots=x^{(n-2)}(0)=0 \\
D_{0^{+}}^{\alpha} x(0)=0, \quad \lim _{t \rightarrow+\infty} D_{0^{+}}^{\alpha-1} x(t)=\int_{0}^{\infty} h(t) x(t) d t
\end{array}\right.
$$

where $0<\beta \leq 1, n-1<\alpha \leq n, n \geq 2, D_{0^{+}}^{\alpha}$ and $D_{0^{+}}^{\beta}$ are standard Riemann-Liouville derivatives, $\varphi_{p}$ is the $p$-Laplacian operator defined by $\varphi_{p}(s)=|s|^{p-2} s,\left(\varphi_{p}\right)^{-1}=\varphi_{q}, \frac{1}{p}+\frac{1}{q}=1, p>1$, and

$$
(T x)(t)=\int_{0}^{t} K(t, s) x(s) d s, \quad(S x)(t)=\int_{0}^{\infty} H(t, s) x(s) d s,
$$

in which $K \in C(D, J), D=\{(t, s) \in J \times J: t \geq s\}, H \in C(J \times J, J), h \in L(J, J)$ with $\int_{0}^{\infty} h(t) \times$ $t^{\alpha-1} d t<\Gamma(\alpha), a \in L(J, J), f \in C(J \times J \times J \times J, J), J=[0,+\infty), J^{\prime}=(0,+\infty)$.

Fractional operators were mentioned by Leibnitz in a letter to L'Hospital in 1695. However, for a quite long period, the theory of fractional derivatives developed mainly as a pure

\section{Springer}

(c) 2015 Wang et al.; licensee Springer. This is an Open Access article distributed under the terms of the Creative Commons Attribution License (http://creativecommons.org/licenses/by/4.0), which permits unrestricted use, distribution, and reproduction in any medium, provided the original work is properly credited. 
theoretical field of mathematics. The situation has changed recently, fractional calculus was shown to be an excellent tool for the description of memory and hereditary properties of various materials and processes. Nowadays, differential equations of fractional order have recently proved to be valuable tools in the modeling of many physical processes, such as non-Markovian diffusion process with memory (see [1]), charge transport in amorphous semiconductors (see [2]), propagation of mechanical waves in viscoelastic media (see [3]), etc. Moreover, phenomena in aerodynamics, electrodynamics of a complex medium or polymer rheology, acoustics, and electro chemistry are also described by differential equations of fractional order (see $[4,5])$. For instance, a viscoelastic fluid with the fractional derivative Maxwell model and its constitutive equation is given by [6]

$$
\sigma+\lambda^{\alpha} \frac{d^{\alpha} \sigma}{d t^{\alpha}}=G \lambda^{\beta} \frac{d^{\beta} \varepsilon}{d t^{\beta}}
$$

where $\sigma$ is the shear stress, $\varepsilon$ is the shear strain, $\lambda=\mu / G$ is the relaxation time, $G$ is the shear modulus, $\mu$ is the viscosity constant, $\alpha$ and $\beta$ are fractional calculus parameters and satisfy $0 \leq \alpha \leq \beta \leq 1$.

Motivated by the fractional calculus' application background, there are a large number of papers dealing with the solvability of fractional differential equations (see [7-12]). By using the Leray-Schauder nonlinear alternative theorem, Zhao and Ge in [13] obtained some results as regards the existence of unbounded solutions by considering the fractional order differential equation

$$
\left\{\begin{array}{l}
D_{0^{+}}^{\alpha} u(t)+f(t, u(t))=0, \quad t \in J^{\prime} \\
u(0)=0, \quad \lim _{t \rightarrow+\infty} D_{0^{+}}^{\alpha-1} u(t)=\beta u(\xi)
\end{array}\right.
$$

where $1<\alpha \leq 2, D_{0^{+}}^{\alpha}$ is the standard Riemann-Liouville fractional derivative and $0<\xi<$ $+\infty, \beta>0, \beta \xi^{\alpha-1}<\Gamma(\alpha), f \in C(J \times \mathbb{R}, J), \mathbb{R}=(-\infty,+\infty)$.

In [14], Liang and Zhang investigated the following $m$-point fractional boundary value problem (BVP) on infinite intervals:

$$
\left\{\begin{array}{l}
D_{0^{+}}^{\alpha} u(t)+a(t) f(u(t))=0, \quad t \in J^{\prime}, \\
u(0)=u^{\prime}(0)=0, \quad \lim _{t \rightarrow+\infty} D_{0^{+}}^{\alpha-1} u(t)=\sum_{i=1}^{m-2} \beta_{i} u\left(\xi_{i}\right)
\end{array}\right.
$$

where $2<\alpha \leq 3, D_{0^{+}}^{\alpha}$ is the standard Riemann-Liouville fractional derivative and $0<$ $\xi_{1}<\xi_{2}<\cdots<\xi_{m-2}<+\infty, \beta_{i}>0$ satisfies $0<\sum_{i=1}^{m-2} \beta_{i} u\left(\xi_{i}\right)<\Gamma(\alpha), a \in L(J, J), f \in C(J, J)$. Through the use of the fixed point index theory due to Leggett-Williams, the sufficient conditions for the existence of three positive solutions are obtained.

Chai in [15] studied the fractional boundary value problem with $p$-Laplacian operator

$$
\left\{\begin{array}{l}
D_{0^{+}}^{\beta}\left(\varphi_{p}\left(D_{0^{+}}^{\alpha} u(t)\right)\right)+f(t, u(t))=0, \quad 0<t<1 \\
u(0)=0, \quad u(1)+\sigma D_{0^{+}}^{\gamma} u(1)=0, \quad D_{0^{+}}^{\alpha-1} u(0)=0
\end{array}\right.
$$

where $1<\alpha \leq 2,0<\beta, \gamma \leq 1,0 \leq \alpha-\gamma-1, D_{0^{+}}^{\alpha}, D_{0^{+}}^{\beta}$, and $D_{0^{+}}^{\gamma}$ are standard RiemannLiouville derivatives, $\sigma$ is a positive constant, $\varphi_{p}$ is the $p$-Laplacian operator defined by $\varphi_{p}(s)=|s|^{p-2} s,\left(\varphi_{p}\right)^{-1}=\varphi_{q}, \frac{1}{p}+\frac{1}{q}=1, p>1, f \in C([0,1] \times J, J)$. By applying the fixed point 
theorem of Leggett-Williams, the author in [15] acquired the existence of positive solutions.

Since the existence of positive solutions to fractional boundary value problems with $p$-Laplacian operator have been rarely researched, in this paper, we investigate the existence of solutions for the fractional differential equation with $p$-Laplacian operator on infinite intervals as the BVP (1.1). We should mention here that our work presented in this paper has various new features. Firstly, the positive solutions on $J$ are obtained, which expands the domain of definition of $t$ from a finite interval to an infinite interval. Secondly, the new terms Tu, Su added in the function $f$ of BVP (1.1) and the more general boundary conditions make the equation we discuss more complicated than those of twopoint, three-point, multi-point boundary conditions. Finally, through the monotone iterative technique, we not only obtain the maximal and minimal solutions to the fractional differential equation but also establish iterative schemes for approximating the solutions, which start from the known simple linear functions.

\section{Preliminaries and lemmas}

Definition 2.1 Let $(E,\|\cdot\|)$ be a real Banach space. A nonempty, closed, convex set $P \subset E$ is said to be a cone provided the following are satisfied:

(a) If $y \in P$ and $\lambda>0$, then $\lambda y \in P$.

(b) If $y \in P$ and $-y \in P$, then $y=0$.

If $P \subset E$ is a cone, we denote the order induced by $P$ on $E$ by $\leq$, that is, $x \leq y$ if and only if $y-x \in P$.

Definition 2.2 [16,17] Let $\alpha>0$ and let $u$ be piecewise continuous on $J^{\prime}$ and integrable on any finite subinterval of $J$. Then for $t>0$, we call

$$
I_{0^{+}}^{\alpha} u(t)=\frac{1}{\Gamma(\alpha)} \int_{0}^{t}(t-s)^{\alpha-1} u(s) d s,
$$

the Riemann-Liouville fractional integral of $u$ of order $\alpha$.

Definition 2.3 $[16,17]$ The Riemann-Liouville fractional derivative of order $\alpha>0, n-1 \leq$ $\alpha<n, n \in \mathbb{N}$, is defined as

$$
D_{0^{+}}^{\alpha} u(t)=\frac{1}{\Gamma(n-\alpha)}\left(\frac{d}{d t}\right)^{n} \int_{0}^{t}(t-s)^{n-\alpha-1} u(s) d s
$$

where $\mathbb{N}$ denotes the natural number set, the function $u(t)$ is $n$ times continuously differentiable on $J$.

Lemma 2.1 $[16,17]$ Let $\alpha>0$, if the fractional derivative $D_{0^{+}}^{\alpha-1} u(t)$ and $D_{0^{+}}^{\alpha} u(t)$ are continuous on $J$, then

$$
I_{0^{+}}^{\alpha} D_{0^{+}}^{\alpha} u(t)=u(t)+c_{1} t^{\alpha-1}+c_{2} t^{\alpha-2}+\cdots+c_{n} t^{\alpha-n},
$$

where $c_{1}, c_{2}, \ldots, c_{n} \in R, n$ is the smallest integer greater than or equal to $\alpha$. 
By a similar proof to Lemma 2.3 in [18], we get Lemma 2.2.

Lemma 2.2 Let $y \in C(0,+\infty) \cap L[0,+\infty)$, then the fractional BVP

$$
\left\{\begin{array}{l}
D_{0^{+}}^{\alpha} x(t)+y(t)=0, \quad t \in J^{\prime}, n-1<\alpha \leq n, n \geq 2, \\
x(0)=x^{\prime}(0)=\cdots=x^{(n-2)}=0, \quad \lim _{t \rightarrow+\infty} D_{0^{+}}^{\alpha-1} x(t)=\int_{0}^{\infty} h(t) x(t) d t,
\end{array}\right.
$$

has a unique solution

$$
x(t)=\int_{0}^{\infty} G(t, s) y(s) d s,
$$

where

$$
G(t, s)=G_{0}(t, s)+G_{1}(t, s)
$$

and

$$
\begin{aligned}
& G_{0}(t, s)=\frac{1}{\Gamma(\alpha)} \begin{cases}t^{\alpha-1}-(t-s)^{\alpha-1}, & 0 \leq s \leq t \leq+\infty, \\
t^{\alpha-1}, & 0 \leq t \leq s \leq+\infty,\end{cases} \\
& G_{1}(t, s)=\frac{t^{\alpha-1}}{\Gamma(\alpha)-\int_{0}^{\infty} h(t) t^{\alpha-1} d t} \int_{0}^{\infty} h(t) G_{0}(t, s) d t .
\end{aligned}
$$

Lemma 2.3 The Green function $G(t, s)$ defined as (2.1) in Lemma 2.2 has the following properties:

(1) $G(t, s)$ is continuous and $G(t, s) \geq 0$ for $(t, s) \in J \times J$.

(2) $\frac{G_{0}(t, s)}{1+t^{\alpha-1}} \leq \frac{1}{\Gamma(\alpha)}, \frac{G(t, s)}{1+t^{\alpha-1}} \leq L$, for $(t, s) \in J \times J$, where $L=\frac{1}{\Gamma(\alpha)-\int_{0}^{\infty} h(t) t^{\alpha-1} d t}$.

Now, we consider the associated linear BVP

$$
\left\{\begin{array}{l}
D_{0^{+}}^{\beta}\left(\varphi_{p}\left(D_{0^{+}}^{\alpha} x(t)\right)\right)+y(t)=0, \quad t \in J^{\prime}, 0<\beta \leq 1, n-1<\alpha \leq n, n \geq 2, \\
x(0)=x^{\prime}(0)=\cdots=x^{(n-2)}=0, \quad D_{0^{+}}^{\alpha} x(0)=0, \\
\lim _{t \rightarrow+\infty} D_{0^{+}}^{\alpha-1} x(t)=\int_{0}^{\infty} h(t) x(t) d t,
\end{array}\right.
$$

where $y \in C(0,+\infty) \cap L[0,+\infty)$ with $\int_{0}^{\infty} \varphi_{q}\left(\int_{0}^{s}(s-\tau)^{\beta-1} y(\tau) d \tau\right) d s<+\infty$. For convenience, let $\omega=(\Gamma(\beta))^{1-q}$, then we have Lemma 2.4.

Lemma 2.4 The associated linear BVP (2.2) has the unique positive solution

$$
x(t)=\omega \int_{0}^{\infty} G(t, s) \varphi_{q}\left(\int_{0}^{s}(s-\tau)^{\beta-1} y(\tau) d \tau\right) d s .
$$

Proof By Lemma 2.1, we have

$$
\varphi_{p}\left(D_{0^{+}}^{\alpha} x(t)\right)=c t^{\beta-1}-\int_{0}^{t} \frac{(t-s)^{\beta-1} y(s)}{\Gamma(\beta)} d s .
$$

Together with the fact $D_{0^{+}}^{\alpha} x(0)=0$, we get $c=0$, then

$$
D_{0^{+}}^{\alpha} x(t)+\varphi_{q}\left(\int_{0}^{t} \frac{(t-s)^{\beta-1} y(s)}{\Gamma(\beta)} d s\right)=0 .
$$


Therefore, BVP (2.2) is equivalent to the following BVP:

$$
\left\{\begin{array}{l}
D_{0^{+}}^{\alpha} x(t)+\varphi_{q}\left(\int_{0}^{t} \frac{(t-s)^{\beta-1} y(s)}{\Gamma(\beta)} d s\right)=0, \quad t \in J^{\prime}, 0<\beta \leq 1, n-1<\alpha \leq n, n \geq 2, \\
x(0)=x^{\prime}(0)=\cdots=x^{(n-2)}=0, \quad \lim _{t \rightarrow+\infty} D_{0^{+}}^{\alpha-1} x(t)=\int_{0}^{\infty} h(t) x(t) d t .
\end{array}\right.
$$

By Lemma 2.2, BVP (2.2) is equivalent to the integral equation (2.3). This completes the proof of the lemma.

In this paper, the following space $E$ will be used in the study of BVP (1.1), where

$$
E=\left\{x \in C[0,+\infty): \sup _{t \in J} \frac{|x(t)|}{1+t^{\alpha-1}}<+\infty\right\}
$$

Then $E$ is a Banach space equipped with the norm $\|x\|=\sup _{t \in J} \frac{|x(t)|}{1+t^{\alpha-1}}$. Define the cone $K \subset E$ by

$$
K=\{x \in E: x(t) \geq 0, t \in J\}
$$

Throughout this paper, we assume the following conditions hold:

$\left(\mathrm{H}_{1}\right)$

$$
\begin{aligned}
& \sup _{t \in J} \frac{1}{1+t^{\alpha-1}} \int_{0}^{t} K(t, s)\left(1+s^{\alpha-1}\right) d s<+\infty \\
& \sup _{t \in J} \frac{1}{1+t^{\alpha-1}} \int_{0}^{\infty} H(t, s)\left(1+s^{\alpha-1}\right) d s<+\infty, \\
& \lim _{t^{\prime} \rightarrow t} \int_{0}^{\infty}\left|H\left(t^{\prime}, s\right)-H(t, s)\right|\left(1+s^{\alpha-1}\right) d s=0, \quad t, t^{\prime} \in J .
\end{aligned}
$$

In this case, let

$$
\begin{aligned}
& k^{*}=\sup _{t \in J} \frac{1}{1+t^{\alpha-1}} \int_{0}^{t} K(t, s)\left(1+s^{\alpha-1}\right) d s, \\
& h^{*}=\sup _{t \in J} \frac{1}{1+t^{\alpha-1}} \int_{0}^{\infty} H(t, s)\left(1+s^{\alpha-1}\right) d s .
\end{aligned}
$$

$\left(\mathrm{H}_{2}\right) f \in C(J \times J \times J \times J, J), f(t, 0,0,0,0) \not \equiv 0, t \in J$, and $f\left(t,\left(1+t^{\alpha-1}\right) u_{0},\left(1+t^{\alpha-1}\right) u_{1},(1+\right.$ $\left.\left.t^{\alpha-1}\right) u_{2}\right)$ is bounded, for $t \in J, u_{i} \in D(i=0,1,2), D \subset J$ is a closed bounded subinterval.

$\left(\mathrm{H}_{3}\right) a \in L(J, J), a(t) \not \equiv 0, t \in J$, and

$$
0<\int_{0}^{\infty} a(s) d s<+\infty, \quad 0<\int_{0}^{\infty} \varphi_{q}\left(\int_{0}^{s}(s-\tau)^{\beta-1} a(\tau) d \tau\right) d s<+\infty .
$$

Denote an operator $A: K \rightarrow E$ by

$$
(A x)(t)=\omega \int_{0}^{\infty} G(t, s) \varphi_{q}\left(\int_{0}^{s}(s-\tau)^{\beta-1} a(\tau) f(\tau, x(\tau),(T x)(\tau),(S x)(\tau)) d \tau\right) d s, \quad t \in J
$$

Under the assumptions $\left(\mathrm{H}_{1}\right)-\left(\mathrm{H}_{3}\right), x$ is a positive solutions of BVP (1.1) if and only if $x$ is a fixed point of $A$ in $K$. 
We list the following lemma, which is needed in our study.

Lemma 2.5 $[19,20]$ Let $E$ be defined as $(2.4)$ and $M$ be any bounded subset of $E$. Then $M$ is relatively compact in $E$, if $\left\{\frac{x(t)}{1+t^{\alpha-1}}: x \in M\right\}$ is equicontinuous on any finite subinterval of $J$ and for any given $\varepsilon>0$, there exists a $N>0$, such that $\left|\frac{x\left(t_{1}\right)}{1+t_{1}^{\alpha-1}}-\frac{x\left(t_{2}\right)}{1+t_{1}^{\alpha-1}}\right|<\varepsilon$ uniformly with respect to all $x \in M$, and $t_{1}, t_{2}>N$.

\section{Main results}

Theorem 3.1 Assume that $\left(\mathrm{H}_{1}\right)-\left(\mathrm{H}_{3}\right)$ hold. Then $A: K \rightarrow K$ is a completely continuous operator.

Proof First, by routine discussion, we see that $A: K \rightarrow K$ is well defined. Now, we prove that $A$ is compact and continuous, respectively. Let $M$ be any bounded subset of $K$. Then there exists $R_{1}>0$, such that $\|x\| \leq R_{1}$, for any $x \in M$. So, for any $x \in M$, by Lemma 2.3 , we have

$$
\begin{aligned}
\|(A x)\|= & \sup _{t \in J} \frac{1}{1+t^{\alpha-1}} \mid \omega \int_{0}^{\infty} G(t, s) \\
& \times \varphi_{q}\left(\int_{0}^{s}(s-\tau)^{\beta-1} a(\tau) f(\tau, x(\tau),(T x)(\tau),(S x)(\tau)) d \tau\right) d s \mid \\
\leq & \omega L \varphi_{q}\left(S_{R_{1}}\right) \int_{0}^{\infty} \varphi_{q}\left(\int_{0}^{s}(s-\tau)^{\beta-1} a(\tau) d \tau\right) d s<+\infty
\end{aligned}
$$

where

$$
\begin{aligned}
S_{R_{1}}= & \sup \left\{f\left(t,\left(1+t^{\alpha-1}\right) u_{0},\left(1+t^{\alpha-1}\right) u_{1},\left(1+t^{\alpha-1}\right) u_{2}\right):\right. \\
& \left.\left(t, u_{0}, u_{1}, u_{2}\right) \in J \times\left[0, R_{1}\right] \times\left[0, k^{*} R_{1}\right] \times\left[0, h^{*} R_{1}\right]\right\} .
\end{aligned}
$$

So, $A M$ is bounded in $E$.

Given $b>0$, for any $x \in M$ and $t_{1}, t_{2} \in[0, b]$, without loss of generality, we may assume that $t_{1}<t_{2}$. In fact,

$$
\begin{aligned}
& \left|\frac{(A x)\left(t_{1}\right)}{1+t_{1}^{\alpha-1}}-\frac{(A x)\left(t_{2}\right)}{1+t_{2}^{\alpha-1}}\right| \\
& \leq \omega \int_{0}^{\infty}\left(\left|\frac{G_{0}\left(t_{1}, s\right)}{1+t_{1}^{\alpha-1}}-\frac{G_{0}\left(t_{2}, s\right)}{1+t_{2}^{\alpha-1}}\right|+\frac{\int_{0}^{\infty} h(t) G_{0}(t, s) d t}{\Gamma(\alpha)-\int_{0}^{\infty} h(t) t^{\alpha-1} d t}\left|\frac{t_{1}^{\alpha-1}}{1+t_{1}^{\alpha-1}}-\frac{t_{2}^{\alpha-1}}{1+t_{2}^{\alpha-1}}\right|\right) \\
& \quad \times \varphi_{q}\left(\int_{0}^{s}(s-\tau)^{\beta-1} a(\tau) f(\tau, x(\tau),(T x)(\tau),(S x)(\tau)) d \tau\right) d s \\
& \leq \omega \varphi_{q}\left(S_{R_{1}}\right) \int_{0}^{\infty}\left(\left|\frac{G_{0}\left(t_{1}, s\right)}{1+t_{1}^{\alpha-1}}-\frac{G_{0}\left(t_{2}, s\right)}{1+t_{1}^{\alpha-1}}\right|+\left|\frac{G_{0}\left(t_{2}, s\right)}{1+t_{1}^{\alpha-1}}-\frac{G_{0}\left(t_{2}, s\right)}{1+t_{2}^{\alpha-1}}\right|\right. \\
& \left.\quad+\frac{\int_{0}^{\infty} h(t) G_{0}(t, s) d t}{\Gamma(\alpha)-\int_{0}^{\infty} h(t) t^{\alpha-1} d t}\left|\frac{t_{1}^{\alpha-1}}{1+t_{1}^{\alpha-1}}-\frac{t_{2}^{\alpha-1}}{1+t_{2}^{\alpha-1}}\right|\right) \\
& \quad \times \varphi_{q}\left(\int_{0}^{s}(s-\tau)^{\beta-1} a(\tau) d \tau\right) d s .
\end{aligned}
$$


On the other hand, we have

$$
\begin{aligned}
& \omega \varphi_{q}\left(S_{R_{1}}\right) \int_{0}^{\infty}\left|\frac{G_{0}\left(t_{1}, s\right)}{1+t_{1}^{\alpha-1}}-\frac{G_{0}\left(t_{2}, s\right)}{1+t_{1}^{\alpha-1}}\right| \varphi_{q}\left(\int_{0}^{s}(s-\tau)^{\beta-1} a(\tau) d \tau\right) d s \\
& \leq \omega \varphi_{q}\left(S_{R_{1}}\right)\left(\int_{0}^{t_{1}}+\int_{t_{1}}^{t_{2}}+\int_{t_{2}}^{\infty}\right)\left|\frac{G_{0}\left(t_{1}, s\right)}{1+t_{1}^{\alpha-1}}-\frac{G_{0}\left(t_{2}, s\right)}{1+t_{1}^{\alpha-1}}\right| \varphi_{q}\left(\int_{0}^{s}(s-\tau)^{\beta-1} a(\tau) d \tau\right) d s \\
& \leq \omega \varphi_{q}\left(S_{R_{1}}\right) \int_{0}^{t_{1}}\left|\frac{t_{2}^{\alpha-1}-t_{1}^{\alpha-1}+\left(t_{2}-s\right)^{\alpha-1}-\left(t_{1}-s\right)^{\alpha-1}}{1+t_{1}^{\alpha-1}}\right| \varphi_{q}\left(\int_{0}^{s}(s-\tau)^{\beta-1} a(\tau) d \tau\right) d s \\
& \quad+\omega \varphi_{q}\left(S_{R_{1}}\right) \int_{t_{1}}^{t_{2}}\left|\frac{t_{2}^{\alpha-1}-t_{1}^{\alpha-1}+\left(t_{2}-s\right)^{\alpha-1}}{1+t_{1}^{\alpha-1}}\right| \varphi_{q}\left(\int_{0}^{s}(s-\tau)^{\beta-1} a(\tau) d \tau\right) d s \\
& \quad+\omega \varphi_{q}\left(S_{R_{1}}\right) \int_{t_{2}}^{\infty}\left|\frac{t_{2}^{\alpha-1}-t_{1}^{\alpha-1}}{1+t_{1}^{\alpha-1}}\right| \varphi_{q}\left(\int_{0}^{s}(s-\tau)^{\beta-1} a(\tau) d \tau\right) d s \\
& \leq \omega \varphi_{q}\left(S_{R_{1}}\right) \int_{0}^{b}\left(\left|t_{2}^{\alpha-1}-t_{1}^{\alpha-1}\right|+\left|\left(t_{2}-s\right)^{\alpha-1}-\left(t_{1}-s\right)^{\alpha-1}\right|\right) \\
& \quad \times \varphi_{q}\left(\int_{0}^{s}(s-\tau)^{\beta-1} a(\tau) d \tau\right) d s \\
& \quad+\omega \varphi_{q}\left(S_{R_{1}}\right) \int_{0}^{\infty}\left(2\left|t_{2}^{\alpha-1}-t_{1}^{\alpha-1}\right|+\left(t_{2}-t_{1}\right)^{\alpha-1}\right) \varphi_{q}\left(\int_{0}^{s}(s-\tau)^{\beta-1} a(\tau) d \tau\right) d s .
\end{aligned}
$$

So, for any $\varepsilon>0$, there exists $\delta_{1}>0$, such that for any $t_{1}, t_{2} \in[0, b]$ and $\left|t_{1}-t_{2}\right|<\delta_{1}$, we have

$$
\omega \varphi_{q}\left(S_{R_{1}}\right) \int_{0}^{\infty}\left|\frac{G_{0}\left(t_{1}, s\right)}{1+t_{1}^{\alpha-1}}-\frac{G_{0}\left(t_{2}, s\right)}{1+t_{1}^{\alpha-1}}\right| \varphi_{q}\left(\int_{0}^{s}(s-\tau)^{\beta-1} a(\tau) d \tau\right) d s<\frac{\varepsilon}{3} .
$$

Similar to (3.1), for the above $\varepsilon>0$, there exists $\delta_{2}>0$, such that for any $t_{1}, t_{2} \in[0, b]$ and $\left|t_{1}-t_{2}\right|<\delta_{2}$, we have

$$
\omega \varphi_{q}\left(S_{R_{1}}\right) \int_{0}^{\infty}\left|\frac{G_{0}\left(t_{2}, s\right)}{1+t_{1}^{\alpha-1}}-\frac{G_{0}\left(t_{2}, s\right)}{1+t_{1}^{\alpha-1}}\right| \varphi_{q}\left(\int_{0}^{s}(s-\tau)^{\beta-1} a(\tau) d \tau\right) d s<\frac{\varepsilon}{3} .
$$

Obviously, for the above $\varepsilon>0$, there exists $\delta_{3}>0$, such that for any $t_{1}, t_{2} \in[0, b]$ and $\mid t_{1}-$ $t_{2} \mid<\delta_{3}$, we have

$$
\begin{aligned}
& \omega \varphi_{q}\left(S_{R_{1}}\right) \int_{0}^{\infty} \frac{\int_{0}^{\infty} h(t) G_{0}(t, s) d t}{\Gamma(\alpha)-\int_{0}^{\infty} h(t) t^{\alpha-1} d t}\left|\frac{t_{1}^{\alpha-1}}{1+t_{1}^{\alpha-1}}-\frac{t_{2}^{\alpha-1}}{1+t_{2}^{\alpha-1}}\right| \\
& \quad \times \varphi_{q}\left(\int_{0}^{s}(s-\tau)^{\beta-1} a(\tau) d \tau\right) d s<\frac{\varepsilon}{3} .
\end{aligned}
$$

So, by (3.1)-(3.3), for the above $\varepsilon>0$, let $\delta=\min \left\{\delta_{1}, \delta_{2}, \delta_{3}\right\}$, such that for any $t_{1}, t_{2} \in[0, b]$ with $\left|t_{1}-t_{2}\right|<\delta$ and for any $x \in M$, we have

$$
\left|\frac{(A x)\left(t_{1}\right)}{1+t_{1}^{\alpha-1}}-\frac{(A x)\left(t_{2}\right)}{1+t_{2}^{\alpha-1}}\right|<\varepsilon
$$

Hence, $\left\{\frac{(A x)(t)}{1+t^{\alpha-1}}: x \in M\right\}$ is equicontinuous on $[0, b]$. Since $b>0$ is arbitrary, $\left\{\frac{(A x)(t)}{1+t^{\alpha-1}}: x \in M\right\}$ is locally equicontinuous on $J$. 
Next, we show that $A: K \rightarrow K$ is equiconvergent at $+\infty$. For any $x \in M$, we have

$$
\begin{aligned}
& \int_{0}^{\infty} \varphi_{q}\left(\int_{0}^{s}(s-\tau)^{\beta-1} a(\tau) f(\tau, x(\tau),(T x)(\tau),(S x)(\tau)) d \tau\right) d s \\
& \quad \leq \varphi_{q}\left(S_{R_{1}}\right) \int_{0}^{\infty} \varphi_{q}\left(\int_{0}^{s}(s-\tau)^{\beta-1} a(\tau) d \tau\right) d s<+\infty
\end{aligned}
$$

and

$$
\begin{aligned}
\lim _{t \rightarrow+\infty} & \frac{|(A x)(t)|}{1+t^{\alpha-1}} \\
= & \lim _{t \rightarrow+\infty} \frac{\omega \int_{0}^{\infty} G(t, s) \varphi_{q}\left(\int_{0}^{s}(s-\tau)^{\beta-1} a(\tau) f(\tau, x(\tau),(T x)(\tau),(S x)(\tau)) d \tau\right) d s}{1+t^{\alpha-1}} \\
= & \lim _{t \rightarrow+\infty} \frac{1}{1+t^{\alpha-1}}\left(\frac{\omega t^{\alpha-1}}{\Gamma(\alpha)} \int_{0}^{\infty} \varphi_{q}\left(\int_{0}^{s}(s-\tau)^{\beta-1} a(\tau) f(\tau, x(\tau),(T x)(\tau),(S x)(\tau)) d \tau\right) d s\right. \\
& -\frac{\omega}{\Gamma(\alpha)} \int_{0}^{t}(t-s)^{\alpha-1} \varphi_{q}\left(\int_{0}^{s}(s-\tau)^{\beta-1} a(\tau) f(\tau, x(\tau),(T x)(\tau),(S x)(\tau)) d \tau\right) d s \\
& +\frac{\omega t^{\alpha-1}}{\Gamma(\alpha)-\int_{0}^{\infty} h(t) t^{\alpha-1} d t} \int_{0}^{\infty} h(t) \int_{0}^{\infty} G_{0}(t, s) \\
& \left.\times \varphi_{q}\left(\int_{0}^{s}(s-\tau)^{\beta-1} a(\tau) f(\tau, x(\tau),(T x)(\tau),(S x)(\tau)) d \tau\right) d s d t\right) \\
= & \frac{\omega}{\Gamma(\alpha)} \int_{0}^{\infty} \varphi_{q}\left(\int_{0}^{s}(s-\tau)^{\beta-1} a(\tau) f(\tau, x(\tau),(T x)(\tau),(S x)(\tau)) d \tau\right) d s \\
& +\frac{\omega}{\Gamma(\alpha)-\int_{0}^{\infty} h(t) t^{\alpha-1} d t} \int_{0}^{\infty} h(t) \int_{0}^{\infty} G_{0}(t, s) \\
& \times \varphi_{q}\left(\int_{0}^{s}(s-\tau)^{\beta-1} a(\tau) f(\tau, x(\tau),(T x)(\tau),(S x)(\tau)) d \tau\right) d s d t \\
\doteq & Q<+\infty
\end{aligned}
$$

So, for any $x \in M$, we have

$$
\begin{aligned}
\left|\frac{(A x)(t)}{1+} t^{\alpha-1}-Q\right| & \\
\leq & \frac{\omega}{\left(1+t^{\alpha-1}\right) \Gamma(\alpha)} \int_{0}^{\infty} \varphi_{q}\left(\int_{0}^{s}(s-\tau)^{\beta-1} a(\tau) f(\tau, x(\tau),(T x)(\tau),(S x)(\tau)) d \tau\right) d s \\
& +\frac{\omega}{\left(1+t^{\alpha-1}\right)\left(\Gamma(\alpha)-\int_{0}^{\infty} h(t) t^{\alpha-1} d t\right)} \\
& \times \int_{0}^{\infty} h(t) \int_{0}^{\infty} G_{0}(t, s) \varphi_{q}\left(\int_{0}^{s}(s-\tau)^{\beta-1} a(\tau) f(\tau, x(\tau),(T x)(\tau),(S x)(\tau)) d \tau\right) d s d t \\
\leq & \frac{\omega \varphi_{q}\left(S_{R_{1}}\right)}{\left(1+t^{\alpha-1}\right) \Gamma(\alpha)} \int_{0}^{\infty} \varphi_{q}\left(\int_{0}^{s}(s-\tau)^{\beta-1} a(\tau) d \tau\right) d s \\
& +\frac{\omega \varphi_{q}\left(S_{R_{1}}\right)}{\left(1+t^{\alpha-1}\right)\left(\Gamma(\alpha)-\int_{0}^{\infty} h(t) t^{\alpha-1} d t\right)} \\
& \times \int_{0}^{\infty} h(t) \int_{0}^{\infty} G_{0}(t, s) \varphi_{q}\left(\int_{0}^{s}(s-\tau)^{\beta-1} a(\tau) d \tau\right) d s d t \\
\rightarrow & 0, t \rightarrow+\infty .
\end{aligned}
$$


Thus, for any $\varepsilon>0$, there exists $N>0$, for any $t>N$ and for any $x \in M$, such that

$$
\left|\frac{(A x)(t)}{1+t^{\alpha-1}}-Q\right|<\frac{\varepsilon}{2}
$$

Consequently, for any $t_{1}, t_{2}>N$ and for any $x \in M$, we have

$$
\left|\frac{(A x)\left(t_{1}\right)}{1+t_{1}^{\alpha-1}}-Q\right|<\frac{\varepsilon}{2}, \quad\left|\frac{(A x)\left(t_{2}\right)}{1+t_{2}^{\alpha-1}}-Q\right|<\frac{\varepsilon}{2} .
$$

Therefore, for any $t_{1}, t_{2}>N$ and for any $x \in M$, we get

$$
\left|\frac{(A x)\left(t_{1}\right)}{1+t_{1}^{\alpha-1}}-\frac{(A x)\left(t_{2}\right)}{1+t_{2}^{\alpha-1}}\right| \leq\left|\frac{(A x)\left(t_{1}\right)}{1+t_{1}^{\alpha-1}}-Q\right|+\left|\frac{(A x)\left(t_{2}\right)}{1+t_{2}^{\alpha-1}}-Q\right|<\varepsilon,
$$

which means that $\left\{\frac{(A x)(t)}{1+t^{\alpha-1}}: x \in M\right\}$ is equiconvergent at $+\infty$. So, $A: K \rightarrow K$ is equiconvergent at $+\infty$.

Finally, suppose $x_{m} \rightarrow x$ as $m \rightarrow+\infty$ in $K$. Then there exists $R_{0}>0$, such that $\max _{m \in \mathbb{N} \backslash\{0\}}\left\{\left\|x_{m}\right\|,\|x\|\right\} \leq R_{0}, \mathbb{N}$ is a natural number set. Since

$$
\begin{aligned}
& \mid \int_{0}^{\infty} \varphi_{q}\left(\int_{0}^{s}(s-\tau)^{\beta-1} a(\tau) f\left(\tau, x_{m}(\tau),\left(T x_{m}\right)(\tau),\left(S x_{m}\right)(\tau)\right) d \tau\right) d s \\
& \quad-\int_{0}^{\infty} \varphi_{q}\left(\int_{0}^{s}(s-\tau)^{\beta-1} a(\tau) f(\tau, x(\tau),(T x)(\tau),(S x)(\tau)) d \tau\right) d s \mid \\
& \quad \leq 2 \varphi_{q}\left(S_{R_{0}}\right) \int_{0}^{\infty} \varphi_{q}\left(\int_{0}^{s}(s-\tau)^{\beta-1} a(\tau) d \tau\right) d s<+\infty
\end{aligned}
$$

where

$$
\begin{aligned}
S_{R_{0}}= & \sup \left\{f\left(t,\left(1+t^{\alpha-1}\right) u_{0},\left(1+t^{\alpha-1}\right) u_{1},\left(1+t^{\alpha-1}\right) u_{2}\right):\right. \\
& \left.\left(t, u_{0}, u_{1}, u_{2}\right) \in J \times\left[0, R_{0}\right] \times\left[0, k^{*} R_{0}\right] \times\left[0, h^{*} R_{0}\right]\right\} .
\end{aligned}
$$

For

$$
\begin{aligned}
& \mid \varphi_{q}\left(\int_{0}^{s}(s-\tau)^{\beta-1} a(\tau) f\left(\tau, x_{m}(\tau),\left(T x_{m}\right)(\tau),\left(S x_{m}\right)(\tau)\right) d \tau\right) \\
& \quad-\varphi_{q}\left(\int_{0}^{s}(s-\tau)^{\beta-1} a(\tau) f(\tau, x(\tau),(T x)(\tau),(S x)(\tau)) d \tau\right) \mid \rightarrow 0, \quad m \rightarrow+\infty .
\end{aligned}
$$

By the Lebesgue dominated convergence theorem, we have

$$
\begin{aligned}
& \mid \int_{0}^{\infty} \varphi_{q}\left(\int_{0}^{s}(s-\tau)^{\beta-1} a(\tau) f\left(\tau, x_{m}(\tau),\left(T x_{m}\right)(\tau),\left(S x_{m}\right)(\tau)\right) d \tau\right) d s \\
& \quad-\int_{0}^{\infty} \varphi_{q}\left(\int_{0}^{s}(s-\tau)^{\beta-1} a(\tau) f(\tau, x(\tau),(T x)(\tau),(S x)(\tau)) d \tau\right) d s \mid \\
& \quad \rightarrow 0, \quad m \rightarrow+\infty
\end{aligned}
$$


Therefore, by Lemma 2.3, we have

$$
\begin{aligned}
& \left\|\left(A x_{m}\right)-(A x)\right\| \\
& =\sup _{t \in J} \frac{1}{1+t^{\alpha-1}} \mid \omega \int_{0}^{\infty} G(t, s) \\
& \quad \times \varphi_{q}\left(\int_{0}^{s}(s-\tau)^{\beta-1} a(\tau) f\left(\tau, x_{m}(\tau),\left(T_{m} x\right)(\tau),\left(S_{m} x\right)(\tau)\right) d \tau\right) d s \\
& \quad-\omega \int_{0}^{\infty} G(t, s) \varphi_{q}\left(\int_{0}^{s}(s-\tau)^{\beta-1} a(\tau) f(\tau, x(\tau),(T x)(\tau),(S x)(\tau)) d \tau\right) d s \mid \\
& \leq \omega L \mid \int_{0}^{\infty} \varphi_{q}\left(\int_{0}^{s}(s-\tau)^{\beta-1} a(\tau) f\left(\tau, x_{m}(\tau),\left(T x_{m}\right)(\tau),\left(S x_{m}\right)(\tau)\right) d \tau\right) d s \\
& \quad-\int_{0}^{\infty} \varphi_{q}\left(\int_{0}^{s}(s-\tau)^{\beta-1} a(\tau) f(\tau, x(\tau),(T x)(\tau),(S x)(\tau)) d \tau\right) d s \mid \\
& \rightarrow 0, \quad m \rightarrow+\infty .
\end{aligned}
$$

Thus, $A: K \rightarrow K$ is continuous.

In conclusion, by Lemma 2.5 , together with the continuity of $A$, we see that $A: K \rightarrow K$ is a completely continuous operator. The proof is completed.

Theorem 3.2 Assume that $\left(\mathrm{H}_{1}\right)-\left(\mathrm{H}_{3}\right)$ hold, and there exists $d>0$ satisfying the following conditions:

$\left(\mathrm{H}_{4}\right) f\left(t, u_{0}, u_{1}, u_{2}\right) \leq f\left(t, \bar{u}_{0}, \bar{u}_{1}, \bar{u}_{2}\right)$, for any $t \in J, 0 \leq u_{0} \leq \bar{u}_{0}, 0 \leq u_{1} \leq \bar{u}_{1}, 0 \leq u_{2} \leq \bar{u}_{2}$.

$\left(\mathrm{H}_{5}\right) f\left(t,\left(1+t^{\alpha-1}\right) u_{0},\left(1+t^{\alpha-1}\right) u_{1},\left(1+t^{\alpha-1}\right) u_{2}\right) \leq \varphi_{p}\left(\frac{d}{\varrho}\right),\left(t, u_{0}, u_{1}, u_{2}\right) \in J \times[0, d] \times\left[0, k^{*} d\right] \times$ $\left[0, h^{*} d\right]$, where

$$
\varrho=\omega L \int_{0}^{\infty} \varphi_{q}\left(\int_{0}^{s}(s-\tau)^{\beta-1} a(\tau) d \tau\right) d s, \quad \text { L is defined by Lemma 2.3. }
$$

Then BVP (1.1) has the maximal and minimal positive solutions $w^{*}, v^{*}$ on J, such that

$$
0<\sup _{t \in J} \frac{\left|w^{*}(t)\right|}{1+t^{\alpha-1}} \leq d, \quad 0<\sup _{t \in J} \frac{\left|v^{*}(t)\right|}{1+t^{\alpha-1}} \leq d
$$

Moreover, for initial values $w_{0}(t)=d t^{\alpha-1}, v_{0}(t)=0, t \in J$, define the iterative sequences $\left\{w_{n}\right\}$ and $\left\{v_{n}\right\}$ by

$$
\begin{aligned}
& w_{n}=\omega \int_{0}^{\infty} G(t, s) \varphi_{q}\left(\int_{0}^{s}(s-\tau)^{\beta-1} a(\tau) f\left(\tau, w_{n-1}(\tau),\left(T w_{n-1}\right)(\tau),\left(S w_{n-1}\right)(\tau)\right) d \tau\right) d s, \\
& v_{n}=\omega \int_{0}^{\infty} G(t, s) \varphi_{q}\left(\int_{0}^{s}(s-\tau)^{\beta-1} a(\tau) f\left(\tau, v_{n-1}(\tau),\left(T v_{n-1}\right)(\tau),\left(S v_{n-1}\right)(\tau)\right) d \tau\right) d s
\end{aligned}
$$

then

$$
\lim _{n \rightarrow+\infty} \sup _{t \in J} \frac{\left|w_{n}(t)-w^{*}(t)\right|}{1+t^{\alpha-1}}=0, \quad \lim _{n \rightarrow+\infty} \sup _{t \in J} \frac{\left|v_{n}(t)-v^{*}(t)\right|}{1+t^{\alpha-1}}=0 .
$$


Proof By Theorem 3.1, $A: K \rightarrow K$ is completely continuous. For any $x_{1}, x_{2} \in K$ with $x_{1} \leq$ $x_{2}$, from the definition of $A$ and $\left(\mathrm{H}_{4}\right)$, we know $A x_{1} \leq A x_{2}$. Let $K_{d}=\{x \in K:\|x\| \leq d\}$. In what follows, we firstly prove $A: K_{d} \rightarrow K_{d}$. In fact, for any $x \in K_{d}$, we have

$$
0 \leq \frac{x(t)}{1+t^{\alpha-1}} \leq d, \quad 0 \leq \frac{(T x)(t)}{1+t^{\alpha-1}} \leq k^{*} d, \quad 0 \leq \frac{(S x)(t)}{1+t^{\alpha-1}} \leq h^{*} d, \quad t \in J .
$$

By $\left(\mathrm{H}_{5}\right)$, we have

$$
f\left(t, u_{0}, u_{1}, u_{2}\right) \leq \varphi_{p}\left(\frac{d}{\varrho}\right), \quad\left(t, u_{0}, u_{1}, u_{2}\right) \in J \times[0, d] \times\left[0, k^{*} d\right] \times\left[0, h^{*} d\right] .
$$

By Lemma 2.3 and $\left(\mathrm{H}_{5}\right)$, we have

$$
\begin{aligned}
\|(A x)\|= & \sup _{t \in J} \frac{1}{1+t^{\alpha-1}} \omega \int_{0}^{\infty} G(t, s) \\
& \times \varphi_{q}\left(\int_{0}^{s}(s-\tau)^{\beta-1} a(\tau) f(\tau, x(\tau),(T x)(\tau),(S x)(\tau)) d \tau\right) d s \\
\leq & \omega L \int_{0}^{\infty} \varphi_{q}\left(\int_{0}^{s}(s-\tau)^{\beta-1} a(\tau) f(\tau, x(\tau),(T x)(\tau),(S x)(\tau)) d \tau\right) d s \\
\leq & \omega L \int_{0}^{\infty} \varphi_{q}\left(\int_{0}^{s}(s-\tau)^{\beta-1} a(\tau) \varphi_{p}\left(\frac{d}{\varrho}\right) d \tau\right) d s=d .
\end{aligned}
$$

Hence, $A: K_{d} \rightarrow K_{d}$.

Let $w_{0}(t)=d t^{\alpha-1}, t \in J$, then $w_{0}(t) \in K_{d}$. Let $w_{1}=A w_{0}, w_{2}=A w_{1}=A^{2} w_{0}$, by Theorem 3.1, we have $w_{1}, w_{2} \in K_{d}$. Denote $w_{n+1}=A w_{n}=A^{n} w_{0}, n=1,2, \ldots$. Since $A: K_{d} \rightarrow K_{d}$, we have $w_{n} \in A\left(K_{d}\right) \subset K_{d}$. It follows from the complete continuity of $A$ that $\left\{w_{n}\right\}_{n=1}^{\infty}$ is a sequentially compact set in $E$. By $(2.1)$ and $\left(\mathrm{H}_{5}\right)$, we have

$$
\begin{aligned}
w_{1}(t)= & \omega \int_{0}^{\infty} G(t, s) \varphi_{q}\left(\int_{0}^{s}(s-\tau)^{\beta-1} a(\tau) f\left(\tau, w_{0}(\tau),\left(T w_{0}\right)(\tau),\left(S w_{0}\right)(\tau)\right) d \tau\right) d s \\
\leq & \frac{\omega t^{\alpha-1}}{\Gamma(\alpha)} \int_{0}^{\infty} \varphi_{q}\left(\int_{0}^{s}(s-\tau)^{\beta-1} a(\tau) f\left(\tau, w_{0}(\tau),\left(T w_{0}\right)(\tau),\left(S w_{0}\right)(\tau)\right) d \tau\right) d s \\
& +\frac{\omega t^{\alpha-1} \int_{0}^{\infty} h(t) t^{\alpha-1} d t}{\Gamma(\alpha)\left(\Gamma(\alpha)-\int_{0}^{\infty} h(t) t^{\alpha-1} d t\right)} \\
& \times \int_{0}^{\infty} \varphi_{q}\left(\int_{0}^{s}(s-\tau)^{\beta-1} a(\tau) f\left(\tau, w_{0}(\tau),\left(T w_{0}\right)(\tau),\left(S w_{0}\right)(\tau)\right) d \tau\right) d s \\
\leq & \omega L t^{\alpha-1} \int_{0}^{\infty} \varphi_{q}\left(\int_{0}^{s}(s-\tau)^{\beta-1} a(\tau) \varphi_{p}\left(\frac{d}{\varrho}\right) d \tau\right) d s \\
= & d t^{\alpha-1}=w_{0}(t) .
\end{aligned}
$$

So, by (3.4) and $\left(\mathrm{H}_{4}\right)$, we have

$$
w_{2}=A w_{1} \leq A w_{0}=w_{1} .
$$

By induction, we get

$$
w_{n+1} \leq w_{n}, \quad n=1,2, \ldots
$$


Thus, there exists $w^{*} \in K$ such that $w_{n} \rightarrow w^{*}$ as $n \rightarrow+\infty$. Applying the continuity of $A$ and $w_{n+1}=A w_{n}$, we get $A w^{*}=w^{*}$.

On the other hand, let $v_{0}(t)=0, t \in J$, then $v_{0}(t) \in K_{d}$. Let $v_{1}=A v_{0}, v_{2}=A v_{1}=A^{2} v_{0}$, then by Theorem 3.1, we have $v_{1}, v_{2} \in K_{d}$. Denote $v_{n+1}=A v_{n}=A^{n} v_{0}, n=1,2, \ldots$. Since $A: K_{d} \rightarrow K_{d}$, we have $v_{n} \in A\left(K_{d}\right) \subset K_{d}$. It follows from the complete continuity of $A$ that $\left\{v_{n}\right\}_{n=1}^{\infty}$ is a sequentially compact set in $E$. Since $v_{1}=A v_{0} \in K_{d}$, we have

$$
v_{2}=A v_{1} \geq 0
$$

By induction, we get

$$
v_{n+1} \geq v_{n}, \quad n=1,2, \ldots
$$

Thus, there exists $v^{*} \in K$ such that $v_{n} \rightarrow v^{*}$ as $n \rightarrow+\infty$. Applying the continuity of $A$ and $v_{n+1}=A v_{n}$, we get $A v^{*}=v^{*}$.

Now, we are in a position to show that $w^{*}$ and $v^{*}$ are the maximal and minimal positive solutions of BVP (1.1) in $\left(0, d t^{\alpha-1}\right]$. Let $u \in\left(0, d t^{\alpha-1}\right]$ be any solution of BVP (1.1), that is, $A u=u$. Noting that $A$ is nondecreasing and $v_{0}(t)=0 \leq u(t) \leq d t^{\alpha-1}=w_{0}(t)$, then we have $v_{1}(t)=\left(A v_{0}\right)(t) \leq u(t) \leq\left(A w_{0}\right)(t)=w_{1}(t)$, for all $t \in J$. By induction, we have

$$
v_{n} \leq u \leq w_{n}, \quad n=1,2,3, \ldots
$$

Since $w^{*}=\lim _{n \rightarrow+\infty} w_{n}, v^{*}=\lim _{n \rightarrow+\infty} v_{n}$, it follows from (3.4)-(3.8) that

$$
v_{0} \leq v_{1} \leq \cdots v_{n} \leq \cdots \leq v^{*} \leq u \leq w^{*} \leq \cdots \leq w_{n} \leq \cdots \leq w_{1} \leq w_{0} .
$$

Since $f(t, 0,0,0) \not \equiv 0, t \in J$, then the zero function is not the solution of BVP (1.1). Therefore, by (3.9), we know that $w^{*}$ and $v^{*}$ are the maximal and minimal positive solutions of BVP (1.1) in $\left(0, d t^{\alpha-1}\right]$, which can be obtained by the corresponding iterative sequences $w_{n}=A w_{n-1}, v_{n}=A v_{n-1}$. The proof is completed.

Remark 3.1 The iterative schemes in Theorem 3.2 are

$$
\begin{aligned}
& w_{0}(t)=d t^{\alpha-1}, \\
& w_{n}=\omega \int_{0}^{\infty} G(t, s) \varphi_{q}\left(\int_{0}^{s}(s-\tau)^{\beta-1} a(\tau) f\left(\tau, w_{n-1}(\tau),\left(T w_{n-1}\right)(\tau),\left(S w_{n-1}\right)(\tau)\right) d \tau\right) d s, \\
& v_{0}(t)=0 \\
& v_{n}=\omega \int_{0}^{\infty} G(t, s) \varphi_{q}\left(\int_{0}^{s}(s-\tau)^{\beta-1} a(\tau) f\left(\tau, v_{n-1}(\tau),\left(T v_{n-1}\right)(\tau),\left(S v_{n-1}\right)(\tau)\right) d \tau\right) d s,
\end{aligned}
$$

they start with a known simple linear function and the zero function, respectively. This is very convenient in applications. So Theorem 3.2 is very interesting and important.

Remark 3.2 By Theorem 3.2, we note that $w^{*}$ and $v^{*}$ are the maximal and minimal solutions of the BVP (1.1) in $K_{d}$, they may coincide, and then BVP (1.1) has only one solution in $K_{d}$. 


\section{Example}

Now we consider the fractional differential equation with a $p$-Laplacian operator on infinite intervals,

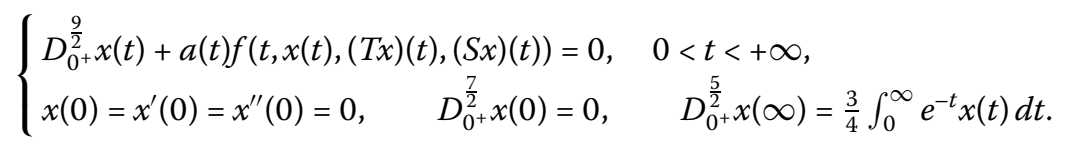

Obviously, $\alpha=\frac{7}{2}, \beta=1, p=2, h(t)=\frac{3}{4} e^{-t}$. By calculation, we have

$$
\Gamma\left(\frac{7}{2}\right)=3.3234, \quad \int_{0}^{\infty} h(t) t^{\alpha-1} d t=2.4925<\Gamma\left(\frac{7}{2}\right) .
$$

Choose

$$
\begin{aligned}
& f\left(t, u_{0}, u_{1}, u_{2}\right) \\
& \quad=10^{-2}\left(\frac{u_{0}}{1+t^{\frac{7}{2}}}\right)^{2}+\frac{10^{-3}}{\left(1+t^{\frac{7}{2}}\right)^{2}}\left(\int_{0}^{t} \frac{u_{1}}{(1+t+s)^{2}\left(1+s^{\frac{7}{2}}\right)} d s+\int_{0}^{\infty} \frac{\cos ^{2}(t-s) u_{2}}{\left(1+s^{2}\right)\left(1+s^{\frac{7}{2}}\right)} d s\right) .
\end{aligned}
$$

So

$$
\begin{aligned}
& K(t, s)=\frac{1}{(1+t+s)^{2}\left(1+s^{\frac{7}{2}}\right)}, \quad H(t, s)=\frac{\cos ^{2}(t-s)}{\left(1+s^{2}\right)\left(1+s^{\frac{7}{2}}\right)}, \\
& \int_{0}^{\infty}\left|H\left(t^{\prime}, s\right)-H(t, s)\right|\left(1+s^{\frac{7}{2}}\right) d s \\
& \quad=\int_{0}^{\infty} \frac{\left|\cos ^{2}\left(t^{\prime}-s\right)-\cos ^{2}(t-s)\right|}{\left(1+s^{2}\right)} d s=\frac{\pi}{2}\left|t^{\prime}-t\right| \rightarrow 0, \quad t^{\prime} \rightarrow t, \\
& k^{*}=\sup _{t \in J} \frac{1}{1+t^{\frac{7}{2}}} \int_{0}^{t} \frac{1+s^{\frac{7}{2}}}{(1+t+s)^{2}\left(1+s^{\frac{7}{2}}\right)} d s=\sup _{t \in J}\left(\frac{1}{(1+t)^{2}}-\frac{1}{(1+t)(1+2 t)}\right) \leq 1, \\
& h^{*}=\sup _{t \in J} \frac{1}{1+t^{\frac{7}{2}}} \int_{0}^{\infty} \frac{\cos ^{2}(t-s)\left(1+s^{\frac{7}{2}}\right)}{\left(1+s^{2}\right)\left(1+s^{\frac{7}{2}}\right)} d s \leq \sup _{t \in J} \frac{1}{1+t^{\frac{7}{2}}} \int_{0}^{\infty} \frac{1}{1+s^{2}} d s=\frac{\pi}{2} .
\end{aligned}
$$

Take $\int_{0}^{\infty} \varphi_{q}\left(\int_{0}^{s} a(\tau) d \tau\right) d s=3$. Considering that

$$
\omega=(\Gamma(\beta))^{1-q}=1, \quad L=\frac{1}{\Gamma(\alpha)-\int_{0}^{\infty} h(t) t^{\alpha-1} d t}=\frac{6}{5},
$$

we get $\varrho=\frac{18}{5}$. Take $d=\sqrt{3}$, then for $\left(t, u_{0}, u_{1}, u_{2}\right) \in J \times[0, d] \times\left[0, k^{*} d\right] \times\left[0, h^{*} d\right]$,

$$
f\left(t,\left(1+t^{\frac{7}{2}}\right) u_{0},\left(1+t^{\frac{7}{2}}\right) u_{1},\left(1+t^{\frac{7}{2}}\right) u_{2}\right) \leq 10^{-2} \cdot 3+\frac{\sqrt{3}}{10^{3}}\left(1+\frac{\pi}{2}\right) \approx 0.03<\varphi_{p}\left(\frac{d}{\varrho}\right) \approx 0.48 .
$$

Thus the conditions in Theorem 3.2 are all satisfied. Therefore, the conclusion of Theorem 3.2 holds. 


\section{Authors' contributions}

The study was carried out in collaboration between all authors. YW completed the main part of this paper and gave two examples; LSL and YHW corrected the main theorems and polished the manuscript. All authors read and approved the final manuscript.

\section{Author details}

${ }^{1}$ School of Mathematical Sciences, Qufu Normal University, Qufu, Shandong 273165, People's Republic of China. ${ }^{2}$ School of Science, Linyi University, Linyi, Shandong 276000, People's Republic of China. ${ }^{3}$ Department of Mathematics and Statistics, Curtin University of Technology, Perth, WA 6845, Australia.

\section{Acknowledgements}

The authors were supported financially by the National Natural Science Foundation of China (11371221), the Specialized Research Foundation for the Doctoral Program of Higher Education of China (20123705110001) and the Program for Scientific Research Innovation Team in Colleges and Universities of Shandong Province.

Received: 14 September 2014 Accepted: 2 January 2015 Published online: 30 January 2015

\section{References}

1. Metzler, R, Klafter, J: Boundary value problems for fractional diffusion equations. Physica A 278, 107-125 (2000)

2. Scher, H, Montroll, EW: Anomalous transit-time dispersion in amorphous solids. Phys. Rev. B 12, 2455-2477 (1975)

3. Mainardi, F: Fractional diffusive waves in viscoelastic solids. In: Wegner, JL, Norwood, FR (eds.) Nonlinear Waves in Solids. ASME/AMR, Fairfield (1995)

4. Gaul, L, Klein, P, Kemple, S: Damping description involving fractional operators. Mech. Syst. Signal Process. 5, 81-88 (1991)

5. Metzler, R, Schick, W, Kilian, HG, Nonnenmacher, TF: Relaxation in filled polymers: a fractional calculus approach. J. Chem. Phys. 103, 7180-7186 (1995)

6. Hilfer, R: Applications of Fractional Calculus in Physics. World Scientific, Singapore (2000)

7. Webb, JRL, Infante, G: Non-local boundary value problems of arbitrary order. J. Lond. Math. Soc. 79, $238-258$ (2009)

8. Chen, TY, Liu, WB, Hu, ZG: A boundary value problem for fractional differential equation with p-Laplacian operator at resonance. Nonlinear Anal. 75, 3210-3217 (2012)

9. Liu, ZH, Lu, L: A class of BVPs for nonlinear fractional differential equations with $p$-Laplacian operator. Electron. J. Qual. Theory Differ. Equ. 2012, 70 (2012)

10. Liu, XP, Jia, M, Xiang, XF: On the solvability of a fractional differential equation model involving the $p$-Laplacian operator. Comput. Math. Appl. 64, 3267-3275 (2012)

11. Liang, SH, Shi, SY: Existence of multiple positive solutions for $m$-point fractional boundary value problems with p-Laplacian operator on infinite interval. J. Appl. Math. Comput. 38, 687-707 (2012)

12. Ding, YZ, Wei, ZL, Xu, JF: Positive solutions for a fractional boundary value problem with $p$-Laplacian operator. J. Appl. Math. Comput. 41, 257-268 (2013)

13. Zhao, XK, Ge, WG: Unbounded solutions for a fractional boundary value problem on the infinite interval. Acta Appl. Math. 109, 495-505 (2010)

14. Liang, $\mathrm{SH}$, Zhang, JH: Existence of three positive solutions of $m$-point boundary value problems for some nonlinear fractional differential equations on an infinite interval. Comput. Math. Appl. 61, 3343-3354 (2011)

15. Chai, GQ: Positive solutions for boundary value problem of fractional differential equation with $p$-Laplacian operator. Bound. Value Probl. 2012, Article ID 18 (2012)

16. Miller, KS, Ross, B: An Introduction to the Fractional Calculus and Fractional Differential Equations. Wiley, New York (1993)

17. Podlubny, I: Fractional Differential Equations. Mathematics in Science and Engineering, vol. 198. Academic Press, New York (1999)

18. Xie, WZ, Luo, ZG, Xiao, J: Successive iteration and positive solutions of a fractional boundary value problem on the half-line. Adv. Differ. Equ. 2013, Article ID 210 (2013)

19. Liu, YS: Boundary value problem for second order differential equations on unbounded domain. Acta Anal. Funct. Appl. 4(3), 211-216 (2002)

20. Corduneanu, C: Integral Equations and Stability of Feedback Systems. Academic Press, New York (1973)

\section{Submit your manuscript to a SpringerOpen ${ }^{\circ}$ journal and benefit from:}

- Convenient online submission

Rigorous peer review

- Immediate publication on acceptance

- Open access: articles freely available online

- High visibility within the field

- Retaining the copyright to your article 